\title{
Recognition and retrieval of objects in diverse applications
}

\author{
Laura Fernández-Robles ${ }^{*+}$ \\ * Johann Bernoulli Institute for Mathematics and Computer Science, University of Groningen, Netherlands \\ ${ }^{+}$Dept. of Electrical, Systems and Automatic Engineering, Universidad de León, Spain \\ Advisors: Enrique Alegre ${ }^{+}$and Manuel Castejón-Limas ${ }^{+}$and Nicolai Petkov* \\ Received 25th Jul 2017; accepted 20th Nov 2017
}

\section{Abstract}

This work proposes and evaluates object description and retrieval techniques in different real applications. First, we addressed the classification of boar spermatozoa according to acrosome integrity, which is an important challenge in the veterinary field. We presented several methods based on invariant local features. We yielded satisfactory results using a concatenation of SURF and global texture descriptors and $k$-NN classification algorithm. Secondly, we focused on the implementation of computer vision solutions for tool wear monitoring, which is a key issue for extending lifetime of cutting tools. We provided two new methods for insert localisation and an automatic solution for the recognition of broken inserts in edge profile milling heads. The proposed approaches are efficient and can be set up in-process without delaying any machining operations. Finally, we worked within the European project Advisory System Against Sexual Exploitation of Children. One of the most challenging tasks in this project was to find specific objects using content-based image retrieval. We evaluated different clusterings of keypoints for object retrieval and proposed a new descriptor, named colour COSFIRE. Colour COSFIRE filters add colour description and improve the discrimination power to COSFIRE filters as well as provide invariance to background intensity.

\section{Motivation}

Object recognition is one of the fundamental tasks in computer vision. This work* studies some particularities of object recognition through three different applications which are highly relevant tasks that pose challenging current computer vision problems on the field of object recognition, see Fig. 1. Firstly, automatic assessment of sperm quality is an important challenge in the veterinary field. In this work, we studied the description of boar spermatozoa acrosomes using image analysis to automatically classify them as intact or damaged. Secondly, wear evaluation of inserts is a key issue for extending lifetime of cutting tools and ensuring high quality of products. In this work, we introduced two image processing methods to automatically localise cutting tools in an edge profile milling head and another one to determine if they are broken. Thirdly, The European project ASASEC aims to provide a technological solution to help the fight against child pornography. One of the most

Correspondence to: Laura Fernández-Robles $<1$. fernandez@unileon.es $>$

Recommended for acceptance by Anjan Dutta and Carles Sánchez

http://dx.doi.org/10.5565/rev/elcvia. 1130

ELCVIA ISSN:1577-5097

Published by Computer Vision Center / Universitat Autònoma de Barcelona, Barcelona, Spain

${ }^{*}$ http://www.rug.nl/research/portal/en/publications/object-recognition-techniques-in-real-applications(a991ab3d-39bb-4a45-b2f4ea8c3a1466a7).html 


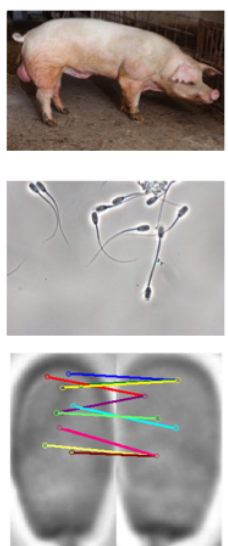

(a)

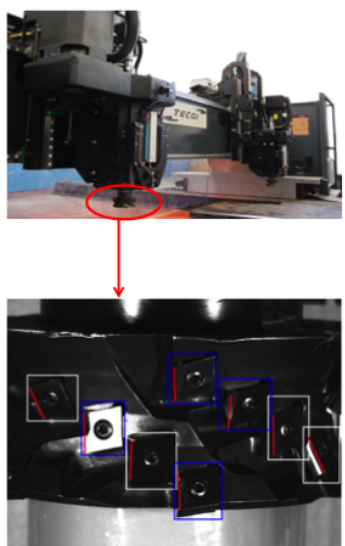

(b)

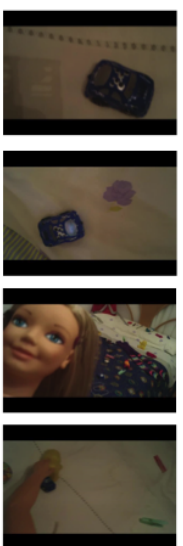

(c)

Figure 1: (a) A boar, a sperm sample and a comparison of the invariant local features of two heads with intact acrosomes. (b) Head of an edge profile milling machine. White rectangles mark intact inserts whereas blue rectangles mark broken ones. Red line segments mark the ideal (intact) cutting edges. (c) Examples of a dataset created to simulate the conditions of ASASEC project, the same blue car object is shown in all images.

challenging tasks in this project was the retrieval of specific objects from collections with a huge amount of images and videos.

\section{Introduction}

In the following, we briefly review the most recent results related with our work for each of the three applications presented. Works dealing with the classification of the acrosome integrity of boar spermatozoa are mainly based on texture description [7]. In this work, we use an invariant local features approach based on the detection and description of keypoints. Many tool wear monitoring systems deal with face milling heads where it is easy to capture one insert per acquired image. In our application, there are 8 to 10 visible inserts per image, which makes the localisation of the inserts a new and challenging task. Some works use the contours of the cutting tools to determine the state of the inserts for drilling or micromilling [8]. We propose a novel algorithm that does not require image references of intact cutting tools. In relation to the last application, COSFIRE filters were introduced by [1] for the localisation of given local patterns that consist of combinations of contour segments. Our main contribution deals with creating a new method, based on COSFIRE filters, that uses of colour information to improve the detection of colour objects and provides an automatic solution for the invariance to background intensity.

\section{Contribution}

There are eight main contributions. 1: We have successfully applied invariant local features, for the first time, in the assessment of sperm acrosome integrity. 2: In the same line of work, we have proposed an approach to classify SURF features, which produce several descriptors per image, with traditional SVM classifiers and without the use of BoW. 3: We have proposed an early fusion of ILF with global texture descriptors for the classification of the integrity of the acrosomes that has outperformed the individual methods. 4: We have presented a highly effective and efficient method for the localisation of cutting edges in milling machines. 5: We have introduced a novel method to describe and classify inserts as broken or unbroken with respect to the state of their cutting edges. 6: We have presented another domain knowledge independent and more versatile method for the localisation of inserts. 7: We have evaluated different clusterings of SIFT keypoints in relation 
with their pose parameters: coordinates location, scale and orientation. 8: We have proposed colour COSFIRE filters that add colour description and improve the discrimination power to standard COSFIRE filters.

\section{Brief methods and results}

\subsection{Classification of boar spermatozoa according to the acrosome integrity}

We characterised the acrosomes using invariant local features, particularly SIFT and SURF, improving the results obtained with global texture descriptors [6]. We achieved the best results using SURF descriptors and the $k$-NN classification algorithm. The overall accuracy was $94.88 \%$, with a higher hit rate for the damaged class $(96.86 \%)$, than for the intact one $(92.89 \%)$. In contrast, we obtained higher hit rates for the intact class using global texture descriptors. In order to classify invariant local features with a support vector machine (SVM), we presented an approach which successfully deals with having more than one descriptor per image [3]. We used SURF to detect and describe interest points. We considered that a spermatozoa head usually contains more keypoints that can be correctly classified than keypoints that could be misclassified. We reached an accuracy of $90.91 \%$ (94.94\% and $86.87 \%$ for the intact and damaged classes respectively) which indicates that this approach could be an alternative to consider for the classification of spermatozoa heads described by invariant local features. We also proposed an early fusion of invariant local features with global texture descriptors to study the integrity of the head acrosomes [3]. We evaluated SVM with bag of visual words (BoW) and $k$-NN for the classification. We achieved an accuracy of $95.56 \%$ (93.63\% in the intact and $97.48 \%$ in the damaged class) using a concatenation of SURF with Legendre descriptors and the $k$-NN classification algorithm. This early fusion is more efficient than the use of the descriptors separately.

\subsection{Automatic localisation of broken inserts in edge profile milling heads}

In this work, we introduced two image processing methods to automatically localise cutting tools in an edge profile milling head and another one to determine if they are broken. Unlike other machining operations presented in the literature, we were dealing with edge milling head tools for aggressive machining of thick plates (up to 12 centimetres) in a single pass. The studied cutting head tool is characterized by its relatively high number of cutting tools (up to 30) which makes the localisation of inserts a key aspect. We detected the screws that fasten the inserts using a circular Hough transform. In a cropped area surrounding a detected screw, we used Canny's algorithm and a standard Hough transform to localise line segments that characterise insert edges. Considering this information and the geometry of the insert, we identified which line segment is the cutting edge. The output of our algorithm is a set of quadrilateral regions around the identified cutting edges that can be used as input to other methods specialised in assessing the state of the cutting edge. Our proposal [4] is very effective (accuracy equals to $99.61 \%$ ) for the localisation of the cutting edges of inserts in an edge profile milling machine. Following up this result, we studied how to recognise broken inserts because it is critical for a proper tool monitoring system [5]. The method that we presented first localises the screws of the inserts and then determines the expected positions and orientations of the cutting edges using known geometrical information. We computed the distances, called deviations, between the expected cutting edge and the real one to determine if it is broken. We evaluated the proposed method on a new dataset that we created and made publicly available. The obtained results, with a F-Score of $91.43 \%$, show that this algorithm is effective and suitable for the recognition of broken inserts in machining head tools. Finally, we proposed a more generic and versatile approach for the localisation of inserts based on trainable COSFIRE filters [3]. It can be automatically configured regardless of the appearance of the inserts. We also introduced a new efficient function for the computation of the response of the COSFIRE filter. We achieved better results, with a F-Score of $89.89 \%$, than preceding works based on template matching. Altogether, the results obtained for this application foster further implementation in a working manufacturing environment. 


\subsection{Object recognition for content-based image retrieval}

We evaluated different clusterings of SIFT keypoints in relation with their pose parameters: coordinates location, scale and orientation [2]. On the one hand, we used the similarity measure of the closest pairs of keypoint descriptors. On the other hand, we used a Hough transform, with different parametrization values, to identify clusters of at least three points voting for the same pose of an object. We verified the consistency of the pose parameters with the least squares algorithm. We computed the results on a publicly available dataset of 614 images that simulates scenes of ASASEC database. We obtained higher precisions at small cuts of the ranked list of retrieved images with the first approach, whereas we yielded better precisions at high cuts with Lowe's clustering. Moreover, we proposed colour COSFIRE filters for the retrieval of colour objects [3]. They add colour description and improve the discrimination power to standard COSFIRE filters as well as provide invariance to background intensity. We presented colour COSFIRE filters both for patterns made up of colour lines and for patterns that are colour objects. Colour COSFIRE filters are more efficient than standard COSFIRE filters both for retrieval and classification tasks.

\section{Conclusion}

This work contributes to the understanding and provides effective solutions of real applications using object recognition and image classification techniques. The proposed computer vision solutions are suitable for the presented problems and they advance the field.

\section{References}

[1] Azzopardi, G., Petkov, N.: Trainable cosfire filters for keypoint detection and pattern recognition. Pattern Analysis and Machine Intelligence, IEEE Transactions on 35(2), 490-503 (Feb 2013)

[2] Fernández-Robles, L., Castejón-Limas, M., Alfonso-Cendón, J., Alegre, E.: Evaluation of clustering configurations for object retrieval using sift features. In: Ayuso Muoz, J.L., Yage Blanco, J.L., Capuz-Rizo, S.F. (eds.) Project Management and Engineering. pp. 279-291. Lecture Notes in Management and Industrial Engineering, Springer International Publishing (2015)

[3] Fernández-Robles, L.: Object recognition techniques in real applications. University of Groningen (2016)

[4] Fernández-Robles, L., Azzopardi, G., Alegre, E., Petkov, N.: Cutting edge localisation in an edge profile milling head. In: Computer Analysis of Images and Patterns, Lecture Notes in Computer Science, vol. 9257, pp. 336-347. Springer International Publishing (2015)

[5] Fernández-Robles, L., Azzopardi, G., Alegre, E., Petkov, N.: Machine-vision-based identification of broken inserts in edge profile milling heads. Robotics and Computer-Integrated Manufacturing 44, $276-283$ (2017)

[6] Fernández-Robles, L., González-Castro, V., García-Olalla, O., García-Ordás, M.T., Alegre, E.: A local invariant features approach for classifying acrosome integrity in boar spermatozoa. In: III Eccomas thematic conference on computational vision and medical image processing (2011)

[7] García-Olalla, O., Alegre, E., Fernández-Robles, L., Malm, P., Bengtsson, E.: Acrosome integrity assessment of boar spermatozoa images using an early fusion of texture and contour descriptors. Computer Methods and Programs in Biomedicine 120(1), 49 - 64 (2015)

[8] Zhang, C., Zhang, J.: On-line tool wear measurement for ball-end milling cutter based on machine vision. Computers in Industry 64(6), 708 - 719 (2013) 